\title{
Many faces, many fields: Social work in changing times
}

As we head towards the general election, we are reminded of the many policies and politics that affect us all as citizens. More than ever, there is a need for us to be careful evaluators of policies that might help or hinder the social justice aims of social work. What policies will decrease poverty? Make housing more affordable and of better quality? Decrease the amount of hospital admissions for preventable childhood illnesses? Deliver funding to health and social services that serve those who need them? Change the demeaning and dehumanising cultures in some of our welfare services? Result in less inequalities? All of these questions are worth considering as we choose who we give our votes to. While we are not here advocating support for any particular party, we like the slogan of one that is "Care. Think. Vote." Social justice and human rights will be at the forefront of your thinking as you enter the polling booth.

Reading academic articles might seem a bit out of touch with urgent practice matters. But it helps us think in new ways about the issues that we care about, and the populations and communities we work with, making our actions as social workers better informed. In this general issue of the journal, there is an array of articles that will hopefully help you "think," covering many diverse topics, as well as a collection of articles focussed on supervision.

Heather Fraser, Nik Taylor and Tania Signal report on a fascinating study into the role of interspecies education in increasing young people's empathy. In their article: "Young people empathising with other animals: Reflections on an Australian RSPCA Humane Education Programme," they show that interspecies education can be a valuable vehicle for promoting empathy amongst young people. Empathy generally promotes pro-social and cooperative behaviour, and is fundamental to social work practice relationships. It is central to the emotional intelligence needed for social work, and can be healing for both givers and receivers of empathic communication. Extending this towards all creatures was an aim of the programme they reported on. The programme they studied was aimed at refugee and migrant young people in Melbourne, a group that contained some young people with limited experiences of positive interactions with animals. After the young people were exposed to animals of various kinds at the RSPCA, and had care for animals modelled to them, they analysed both images of the young people interacting with the animals, and letters they wrote to the organisation following the programme. Quotes are provided to illustrate the themes found in the letters that show the visits shaped the growth of the young people's empathy with animals. For example, Leo noted the differences between his earlier view and the change that occurred following the programme: "I was asking myself why do people like animals? The only thing I was believe is animals are stupid, ugly and always trying to attack the people ... I personally was hate animals ... After came RSPCA I liked animals step by step." The authors argue that more attention to the possibilities of interspecies education to increase empathy, a cornerstone of social work, should be given.

Sally Raymond, Liz Beddoe and Barbara Staniforth's article entitled "Social workers' experiences with whistleblowing: To speak or not to speak?" explores the experiences of whistleblowing of 10 social workers in Aotearoa NZ. The study found that there had been limited support for whistleblowers
AOTEAROA NEW ZEALAND SOCIAL WORK 29(3), 1-4. 
and often retaliation afterwards from colleagues. They note, with some irony, that social workers are expected to engage in advocacy for their service users, but when it comes to organisational practices, whistleblowing, a form of advocacy, can be punished. As one participant noted: “We should be a lot more active. We do it with our clientele and why can't we [advocate for] our staff?" The managerial contexts of practice can be punitive and not open to criticism. The authors conclude that social workers need better information under the Protected Disclosures Act (2000).

In an article with a strong legal analysis, Richard Fisher and Trudy Hutton-Baas make a strong case for improving the legal standing of grandparents caring for their grandchildren. In "Supporting grandparent/ grandchild contact under the Care of Children Act 2004: Assessment and a call for change," they point out that, while the Care of Children Act (COCA) introduced many changes to guardianship status, it did not make any special consideration for grandparents despite the common practice of placing children into their care. This was due to an emphasis at the time on "focusing on the duties, powers, rights and responsibilities of parents as guardians to their children" rather than grandparents; for example, allowing new partners to apply for guardianship but not grandparents. They undertook a policy analysis and an investigation into cases where grandparents litigated in relation to their rights. They conclude there are some helpful measures in the Act and its associated policies, but the fundamental issue of lack of legal standing requires a remedy in order to assure contact can continue. Suggestions are made to adopt legislation more in line with the Canadian model where most provinces have legislation that increases the rights of grandparents relative to other types of family members. There, the issues of grandparents' legal status and human rights legislation are intertwined. As our human rights legislation does not have a "strike down" provision (that would enable judges to invalidate laws that are not consistent with human rights), there is no such impetus for consideration of this here. Fisher points out this means that: "as a result, while there might be a variety of objections a grandparent might raise under the NZBRA to their lack of standing in New Zealand legislation (e.g., freedom from discrimination), there is no scope for a meaningful result by challenging the law on this basis."

In another issue with relevant currency, Deb Stanfield, Liz Beddoe, Neil Ballantyne, Simon Lowe and Nicole Renata report on a study of social workers' perceptions of Facebook use in a professional capacity. In their article, "Critical conversations: Social workers' perceptions of the use of a closed Facebook group as a participatory professional space," they examine social workers' use of a closed online group set up to encourage professional deliberation and public debate about issues related to society and the profession. People in the group were asked via both a survey and interviews, about their motivations for joining and their experiences as members. They found that the reported benefits were access to rapid resource dissemination, the ability to remain connected with current social issues that would otherwise seem "too big" to keep up with, and lessen professional isolation. Problems were also reported, such as a lack of consensus or knowledge about what is ethical behaviour in the online space. Issues relating to insider research also came to the fore in this study and there is thoughtful coverage of this issue.

Kirk Reed and Brian Field provide a challenge in the context of mental health legislation in their article: "Resituating Aotearoa New Zealand mental health legislation in the context of social and occupational justice". In this article, they align social workers with occupational therapists, arguing that they face similar challenges to maintain a focus on justice for their service users in response to the 
dominating "medico-legal worldview." With a strong focus on the historical development of mental health legislation, they argue that the necessities of maintaining a therapeutic relationship and following professional values and beliefs that support a recovery orientation to practice are in conflict with the medical and legal underpinnings of the legislation. They point out that, although the rhetoric of recovery is central to many mental health policies, specifically in the context of compulsory assessment and treatment orders, that social workers and occupational therapists may conflict with other more dominant professionals who may be more risk-averse. While legal and other changes have led to a reduction in psychiatric beds around the country and a greater focus on community provision, legislation still remains in tension with recovery principles, as it suggests that people are so risky that legislation must address this with compulsory treatment orders. They stress that this has serious rights issues such as the deprivation of liberty.

This issue also contains two viewpoint pieces. In the first, Carole Adamson and Luis Arevalo engage in a critical discussion entitled "What do you mean, I'm 'resilient'?" They make important points in relation to this much-used, and perhaps misused, concept. Their key point is that people respond to adverse events in unique ways, personal to themselves, that do not always translate directly into the response of the systems that respond to them. They also note that "an uncritical use of the term can result in its use for political agendas contrary to social work values." They point out that part of that "translation" can result in support of the neoliberal ideals of independence, a resignation to the reduction of community and personal resources, and minimise a focus on social context and the need for social change. Particularly in disaster contexts, people should not be exhorted to pursue some ideal model of resilience but, instead, it is up to them to define for themselves the meaning of their experiences and what resilience might mean for them. Assumptions about resilience can encourage a hands-off approach by both government and other supportive services, and people's felt vulnerabilities can be overlooked, particularly when combined with a culture that in some quarters, valorizes "being staunch." In their concluding comments, they encourage us to "think twice" about the term so readily inserted into lectures or practice, when describing people's responses to stressful or distressing events-these should be both considered in context and linked to the broader political environment, one that may minimise structural disadvantage.

The second half of this journal issue has a focus on supervision with four full articles and a short viewpoint piece. The collation of these articles at this time suggests that supervision research and innovation is in a healthy state in Aotearoa New Zealand. Each article makes a unique contribution to the already solid body of local supervision literature. Three of the articles are empirical and the other two are reports on supervisionfocused development.

First up, Matt Rankine, in "Making the connections: A practice model for reflective supervision," describes a new model of reflective supervision. This model has developed following a theoretical analysis in a qualitative study of social work supervision in community based child and family services. Rankine argues for an approach to social work supervision that is grounded in a "co-constructed partnership between the supervisor and supervisee." The four-layered model supports critical thinking in socio-political and cultural contexts and promotes social justice strategies.

Supervision is changing in response to shifts in the organisation of health and social services and an increasing focus on interprofessionality in professional work in multi-disciplinary settings. Allyson Davys, in “Interprofessional supervision: A matter of 
difference," reports findings from a series of semi-structured interviews with participants in interprofessional supervision relationships. Davys begins with a review of the traditions of social work supervision and identifies the professional and regulatory expectations of supervision for social workers in Aotearoa New Zealand. She then discusses the preliminary findings of a cross-professional study of interprofessional supervision finding that it provides an opportunity whereby social workers can enhance their practice through reflection and critique whilst still meeting professional requirements. The participants in this research approached their supervision as an opportunity for professional growth and learning and showed a willingness to "embrace, grapple with, and enjoy, difference."

Ruth Ford's article, "Implementing staff supervision training in a Corrections environment," describes a project which involved the implementation of in-house supervision and supervision training for programme facilitators. Practitioners and managers wanting to develop and/or improve supervision in their organisation will find much useful material in this article as Ford outlines essential areas for focus on the delivery of a successful programme. Ford's literature review explores published material on developing training and, while it is not empirical, and much of it is deeply contextual, significant and useful themes emerge about underlying principles, delivery and content.

In a time where evidence of value for money dominates discussion of every budget item in health and social services, an article by Allyson Davys with colleagues Janet May, Beverley Burns and Michael O'Connor explores the challenging topic of "Evaluating social work supervision." Davys and colleagues report on a survey of a range of professions in Aotearoa New Zealand. Of 329 survey respondents, 245 were social workers. Most social workers who participated in the survey reported that some kind of review of supervision took place but these used varied methods and were not formal. Almost half offered suggestions for improvement, with more systematic approaches favoured.

Of concern in the findings of this study is a theme of harmful supervision where relationship dynamics were detrimental to an effective supervision relationship. The authors ask if this is the tip of an iceberg, and recommend further investigation of social work supervision. In particular, they question the enduring model of line management supervision which is what most social workers access, where there is unequal power and little choice.

Peer supervision models offer much to address problems that are associated with managerial models of supervision. In a final viewpoint piece, "Zooming in: Social work supervisors using online supervision," Jason Rushton, Jo Hutchings, Karen Shepherd and Jude Douglas describe the development of an online group for supervisors using the Zoom ${ }^{\mathrm{TM}}$ application. The technology proved to be effective for this group to connect and share their supervisory experiences and concerns. The group is non-hierarchical with members sharing facilitation and note taking roles in rotation. The authors share their experiences and hope that others will be encouraged to develop similar groups and share their insights to add to social work supervision expertise in Aotearoa New Zealand.

\section{Liz Beddoe and Emily Keddell}

